\title{
Subtype analysis of Blastocystis spp. isolated from domestic mammals and poultry and its relation to transmission to their in- contact humans in Ismailia governorate, Egypt.
}

\author{
Original \\ Article \\ Amira B. Mokhtar ${ }^{1}$ and Ahmed I. Youssef ${ }^{2}$ \\ ${ }^{1}$ Medical Parasitology Department, Faculty of Medicine, Suez Canal University, Ismailia, \\ 41522, Egypt. \\ ${ }^{2}$ Animal Hygiene and Zoonoses Department, division of zoonoses, Faculty of Veterinary \\ Medicine, Suez Canal University, Ismailia, Egypt.
}

\begin{abstract}
Background: Blastocystis is a common protist detected in fecal samples of humans and a wide range of animals. The parasite exhibits extensive genetic diversity with seventeen distinct subtypes (STs) identified collectively from humans, other mammals and birds. Shared STs between animal and human hosts were considered to be potentially zoonotic. However, Blastocystis infection among non-human hosts, in Egypt has not been investigated so far.

Objective: To determine the occurrence and ST distribution of Blastocystis species isolated from domestic mammals, poultry and their in-contact humans.

Material and Methods: A total of 416 fecal samples from domestic animals (mammals and poultry) as well as their in-contact humans were screened by inoculation into Jones' media. Positive samples were subtyped using seven pairs of ST-specific sequence-tagged-site (STS) primers.

Results: The occurrence of Blastocystis spp. infection was $69.8 \%$ in poultry, $17.7 \%$ in domestic mammals and $35.7 \%$ in humans. Among the studied animal species ST1-ST7 were identified with varying percentages; however, only four STs (ST1-ST4) were identified in humans. A minority of human subjects examined (16/56, 28.5\%) were carrying the same ST detected in their domestic animals.

Conclusion: The detection of all the tested STs among the infected animal species examined highlights the broad genetic diversity observed among Blastocystis spp. isolated from animals. However, the detection of only four STs among humans suggests that these STs can easily infect humans and the animals carrying the same STs could be possible reservoirs. Surprisingly, direct handling of animals was not found to be a major contributor to human blastocystosis in Egypt, denoting the role of anthroponotic transmission and the possibility of fecal cross-contamination from other potential reservoir animals in the surrounding environment.
\end{abstract}

Key Words: Blastocystis, Egypt, humans, mammals, poultry, subtype.

Received: 29 December 2017, Accepted: 14 February 2018.

Corresponding Author: Amira B Mokhtar, Tel.: +201142753199, E-mail: ami_hagag@yahoo.com

ISSN: 1687-7942, Vol. 11, No. 2, August 2018

\section{INTRODUCTION}

Blastocystis is an anaerobic parasite that inhabits the intestinal tract of humans and a wide range of animals such as non-human primates, other mammals, birds, amphibians, reptiles and arthropods ${ }^{[1,2]}$. It is one of the most common eukaryotic organisms detected in human fecal samples worldwide $^{[3]}$. Four main forms of Blastocystis have been described: vacuolar, granular, amoeboid and cyst forms ${ }^{[4,5]}$.

It is transmitted primarily through the fecal-oral route via food or drinks contaminated with the infective cyst stage ${ }^{[6,7]}$. Moreover, waterborne transmission via untreated water or poor sanitary conditions has also been known $^{[8,9]}$. Based on molecular analysis of the smallsubunit rRNA gene, seventeen distinct subtypes (STs) have been identified from humans, non-human primates, other mammals and birds ${ }^{[10]}$. Humans are potential hosts to nine Blastocystis STs (ST1-ST9), where they most frequently host ST3 but are also commonly found to carry ST1, ST2 and ST4 ${ }^{[11,12]}$. The five other STs (ST5-ST9) have been reported sporadically from humans ${ }^{[13-17]}$, with ST9 being reported in human only and not in non-human hosts until now ${ }^{[18]}$.

Several studies have linked human infections with specific Blastocystis STs to potential animal sources, providing evidence to support the parasite's zoonotic potential ${ }^{[12,19-22]}$. Such knowledge is of great importance for epidemiological studies and allows development of control strategies to the parasite's spread ${ }^{[12]}$. The aim of the present study is to investigate the occurrence and the distribution of Blastocystis STs in various non-human hosts (domestic animals) and its role in transmission to in-contact humans 
to understand the epidemiology and zoonotic potential of this parasite in Egypt.

\section{MATERIAL AND METHODS}

Collection of fecal samples: In this descriptive study samples were collected from five different regions of Ismailia Governorate in Egypt, mostly from rural areas (Ismailia, Abu Suweir, Fayed, Tell El Kebir and El Qantara) between the period from January 2015 to June 2016. Upon informed consent, samples were collected concurrently from humans and their domestic animals and transferred on the same day to the laboratory of the Parasitology Department, Faculty of Medicine, Suez Canal University where further processing of the samples was carried out. All the procedures were conducted according to the ethical standards approved by the Human Ethics Committee, Faculty of Medicine, Suez Canal University, Egypt.

Fecal samples from domestic animals: Samples included 130 from cattle, donkeys, horses, camels, rabbits, goats, sheep, dogs and cats and 129 from chicken, ducks, geese, turkeys and pigeons, reared in houses and farms, and also animals from veterinary clinics. These domestic animals were in close contact with humans and were reared close to their dwellings. Samples were collected in plastic bags immediately after deposition by the animals; special care was taken to avoid contamination from soil. On some occasions, rectal fecal samples were collected using a sterile swab when fresh fecal samples were not available or when the samples found were significantly contaminated with soil. Owners of domestic animals were asked about diarrheic symptoms in their animals. In the case of poultry, two to three samples of the same species were selected from the same place because of the large number of birds reared in the same place.

Human fecal samples: A total of 157 human fecal samples were collected from all subjects ( 1 sample/subject) who had close contact with animals (involved in animal feeding, cleaning and/or waste removal). A standardized questionnaire was conducted, covering the demographic data, source of drinking water, occupational experience, lifestyle, current contact with animals, presence of gastrointestinal symptoms and current health status.

Culture of fecal samples: To overcome the rather insensitive nature of direct smear and concentration methods in the detection of Blastocystis spp., in vitro cultivation was performed for each stool sample ${ }^{[22]}$ using Jones' medium $^{[23]}$ supplemented with $10 \%$ horse serum. When the typical vacuolar and/or granular forms were observed in cultures by standard light microscopy, they were judged to be positive for infection. If they were not detected until the seventh day of culture, samples were considered negative. Positive cultures were subsequently cultured for at least two subcultures to remove fecal matters. From the second subculture, Blastocystis forms were isolated by centrifugation at $500 \times \mathrm{g}$ for $10 \mathrm{~min}$. The pellet was then washed with Ringer's solution before centrifugation at $500 \times \mathrm{g}$ for $10 \mathrm{~min}$, and this was repeated twice. The supernatant was discarded, and the pellet was transferred to a sterile Eppendorf tube.

DNA extraction and PCR: Blastocystis genomic DNA was extracted from the cell pellets using DNAzol (Gibco-BRL, USA) according to the manufacturer's protocol. The extracted DNA was then analyzed using seven ST-specific (STS) primer sets described by Yoshikawa et $a l .{ }^{[24]}$ and according to the nomenclature established by Stensvold et al. ${ }^{[25]}$, namely: SB83 for ST1(351 bp), SB340 for ST2 (704 bp), SB227 for ST3 (526 bp), SB337 for ST4 (487 bp), SB336 for ST5 (317 bp), SB332 for ST6 (338 bp), and SB155 for ST7 (650 bp). A final volume of $25 \mu \mathrm{l}$ PCR reaction mixture was prepared from $25 \mathrm{pmol}$ of each primer pair (of one subtype at a time), $12.5 \mu \mathrm{l}$ Quick-Load Taq 2X Master Mix (New England Biolab, UK), $9.5 \mu \mathrm{l}$ nuclease-free water and $1 \mu \mathrm{l}$ of template DNA $(40-50 \mathrm{ng} / \mu \mathrm{l})$. A negative control tube was prepared as previously described in which template DNA was replaced by nuclease-free water. The PCR procedure consisted of an initial denaturing at $94^{\circ} \mathrm{C}$ for $3 \mathrm{~min}$, followed by 30 cycles that included denaturing at $94^{\circ} \mathrm{C}$, annealing at $57^{\circ} \mathrm{C}$ for $30 \mathrm{sec}$ each and extension at $72^{\circ} \mathrm{C}$ for $1 \mathrm{~min}$, then an additional cycle with a 10 min chain elongation at $72^{\circ} \mathrm{C}$. PCR amplification for each primer pair was repeated at least twice for each isolate. The amplified products were electrophoresed on $1.5 \%$ agarose gels (Promega, USA) in Tris-borate-EDTA buffer. Gels were stained with ethidium bromide and were photographed using an ultraviolet gel documentation system (Uvitec, United Kingdom).

Statistical analysis: Collected data were coded and processed using a Microsoft Excel software file and the SPSS (version 16.0, Chicago, SPSS Inc., USA) program for analysis. A descriptive analysis of the percentages was used to investigate Blastocystis spp. occurrence and subtype distribution among the studied population.

\section{RESULTS}

The occurrence of Blastocystsis spp.: A total of 129 samples were collected from poultry, of which 29 samples $(69.8 \%)$ were proven positive for Blastocystis, while out of 130 samples collected from different domestic mammals, 23 samples $(17.7 \%)$ were positive. Some mammal species such as horses, rabbits, sheep, domestic cats and dogs examined were negative for Blastocystis infection. The occurrence of Blastocystis spp. was $72.2 \%$ in cattle, $7.1 \%$ in donkeys, $25 \%$ in camels and $28.6 \%$ in goats (Table 1). All domestic mammals and poultry found positive for Blastocystis spp. appeared healthy and active and did not show diarrhea at the time of sample collection. As for humans, 157 samples were collected from those who have routine contact with these animals, of which 56 samples $(35.7 \%)$ were positive for Blastocystis spp. (Table 1). 
Identification of Blastocystis subtypes: The ST distribution of the isolates from different domestic animals and humans in this study is displayed in table (1). The majority of samples had single ST infection, and only a few had mixed ST infections (MSI) (4/90, 3/23 and 5/56 from poultry, domestic mammals and human samples, respectively). Mixed infections were with two STs except for one isolate from a camel (triple ST infection) (Table 1). Among the five poultry species examined (chicken, ducks, geese, turkeys and pigeons), ST7 predominated (77.8\%), followed by ST6 (17.8\%), ST1 (7.8\%) and ST2 the least $(1.1 \%)$. In contrast, among the four infected domestic mammal species (cattle, donkeys, camels and goats), ST1 predominated $(43.5 \%)$, followed by ST4 $(17.4 \%)$, ST3
(13\%), ST5 (8.7\%), and ST6 and ST7 were equally the least ( $4.3 \%$ each). Six positive samples isolated from cattle were not typed with the used STS primers. Among human samples examined, ST1-ST4 were identified, with a predominance of ST3 (60.7\%), followed by ST1 (35.7\%), ST2 $(7.1 \%)$ and ST4 was the least $(5.4 \%)$ (Table 2 and Figure 1).

Regarding the coincidence of detected STs in humans and their domestic animals, $40 \%$ of humans with ST1 infection ( $8 / 20$ cases) were found to have the same ST as their animals. The coincidence was $0 \%$ for ST2, $14.7 \%(5 / 34$ cases) for ST3 and 100\% (3/3) for ST4 (Table 3).

Table 1: Occurrence of Blastocystis spp. and ST classification among studied poultry, domestic mammals and humans

\begin{tabular}{|c|c|c|c|c|c|c|c|c|c|c|c|c|}
\hline \multirow[b]{2}{*}{ Host } & \multirow[b]{2}{*}{ Scientific name } & \multirow{3}{*}{$\begin{array}{c}\text { Collected } \\
\text { samples } \\
\text { no. }\end{array}$} & \multirow{2}{*}{$\begin{array}{l}\text { Positive } \\
\text { no. (\%) }\end{array}$} & \multicolumn{9}{|c|}{ Blastocystis ST no. } \\
\hline & & & & ST1 & ST2 & ST3 & ST4 & ST5 & ST6 & ST7 & $\begin{array}{l}\text { Mixed } \\
\text { (STs) }\end{array}$ & $\begin{array}{c}\text { Not } \\
\text { typed }\end{array}$ \\
\hline \multicolumn{12}{|c|}{ Domestic birds } & \\
\hline Chicken & Gallus gallus domesticus & 57 & $47(82.5)$ & 3 & - & - & - & - & 8 & 34 & $2(6+7)$ & - \\
\hline Ducks & Anas platyrhynchos & 25 & $25(100)$ & 1 & - & - & - & - & - & 23 & $1(6+7)$ & - \\
\hline Geese & Anseranser & 20 & $5(25)$ & 1 & 1 & - & - & - & 1 & 2 & - & - \\
\hline Turkeys & Meleagris gallopavo & 12 & $6(50)$ & 1 & - & - & - & - & 2 & 2 & $1(1+6)$ & - \\
\hline Pigeons & Columba livia domestica & 15 & $7(46.7)$ & - & - & - & - & - & 1 & 6 & - & - \\
\hline Total & & 129 & $90(69.8)$ & 6 & 1 & - & - & - & 12 & 67 & 4 & - \\
\hline \multicolumn{13}{|c|}{ Domestic mammals } \\
\hline Cattle & Bostaurus & 18 & $13(72.2)$ & 2 & - & 3 & - & 2 & - & - & - & 6 \\
\hline Donkeys & Equus africanus asinus & 14 & $1(7.1)$ & 1 & - & - & - & - & - & - & - & - \\
\hline Horses & Equus ferus caballus & 15 & 0 & - & - & - & - & - & - & - & - & - \\
\hline Camels & Camelus dromedarius & 20 & $5(25)$ & 3 & - & - & - & - & - & - & 1 & - \\
\hline Rabbits & Oryctolagus cuniculus & 12 & 0 & - & & - & - & - & - & - & $\begin{array}{l}(1+4+6) \\
1(4+7)\end{array}$ & - \\
\hline Goats & Capra aegagrus hircus & 14 & $4(28.6)$ & 2 & - & - & 1 & - & - & - & - & - \\
\hline Sheep & Ovisaries & 8 & 0 & - & - & - & - & - & - & - & - & - \\
\hline Dogs & Canis lupus familiaris & 21 & 0 & - & - & - & - & - & - & - & - & - \\
\hline Cats & Felis catus & 8 & 0 & - & - & - & - & - & - & - & 3 & - \\
\hline Total & & 130 & $23(17.7)$ & 8 & - & 3 & 1 & 2 & - & - & $4(1+3)$ & 6 \\
\hline Humans & Homo sapiens & 157 & $56(35.7)$ & 16 & 3 & 29 & 3 & - & - & - & $1(2+3)$ & - \\
\hline
\end{tabular}

Table 2: Collective distribution of Blastocystis STs among the studied poultry, domestic mammals and humans

\begin{tabular}{lccccccccc}
\hline Host & $\begin{array}{c}\text { Total no. } \\
\text { of positive } \\
\text { samples }\end{array}$ & ST1 & ST2 & ST3 & ST4 & ST5 & ST6 & ST7 & Not typed \\
\hline Poultry & 90 & $7(7.8)$ & $1(1.1)$ & 0 & 0 & 0 & $16(17.8)$ & $70(77.8)$ & 0 \\
Mammals & 23 & $10(43.5)$ & 0 & $3(13)$ & $4(17.4)$ & $2(8.7)$ & $1(4.3)$ & $1(4.3)$ & $6(26.1)$ \\
Humans & 56 & $20(35.7)$ & $4(7.1)$ & $34(60.7)$ & $3(5.4)$ & 0 & 0 & 0 & 0 \\
\hline
\end{tabular}

*The number includes isolates with MSI 
Table 3: Coincidence of detected Blastocystis STs in humans and their domestic animals

\begin{tabular}{|c|c|c|c|c|c|c|c|c|}
\hline \multirow{3}{*}{$\begin{array}{l}\text { Similar ST detected in domestic } \\
\text { animals }\end{array}$} & \multicolumn{8}{|c|}{ ST detected in humans $(n=56) *$} \\
\hline & \multicolumn{2}{|c|}{ ST1 $(n=20)$} & \multicolumn{2}{|c|}{ ST2 $(n=4)$} & \multicolumn{2}{|c|}{ ST3 $(n=34)$} & \multicolumn{2}{|c|}{ ST4 $(n=3)$} \\
\hline & No. & $\%$ & No. & $\%$ & No. & $\%$ & No. & $\%$ \\
\hline Yes & 8 & 40 & 0 & 0 & 5 & 14.7 & 3 & 100 \\
\hline No & 12 & 60 & 4 & 100 & 29 & 85.3 & 0 & 0 \\
\hline
\end{tabular}

* The number includes isolates with MSI
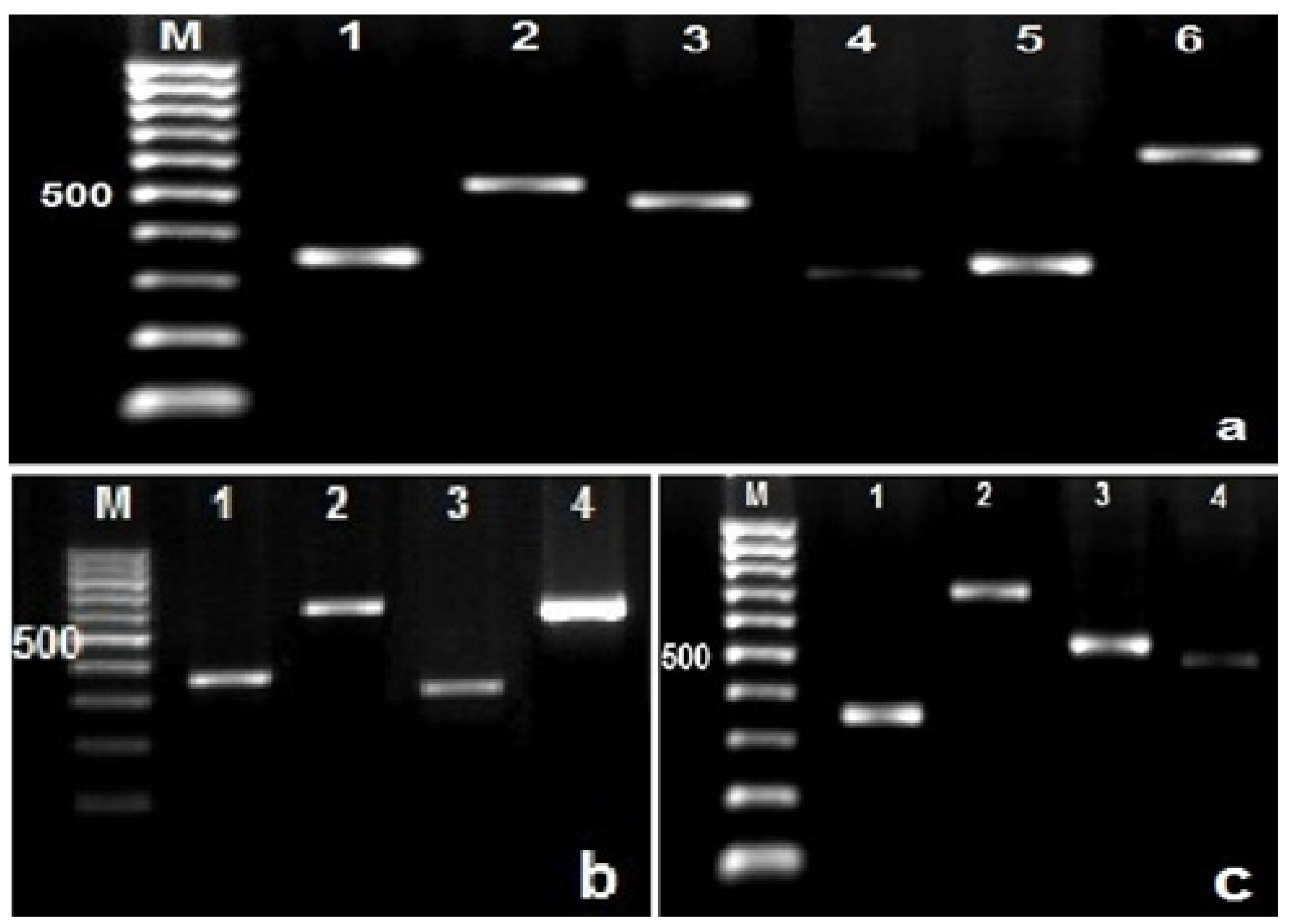

Fig. 1: Examples of PCR products of different Blastocystis STs detected: a) in domestic mammals showing positive bands of ST1 (351 bp) in lane 1, ST3 (526 bp) in lane 2, ST4 (487 bp) in lane 3, ST5 (317 bp) in lane 4, ST6 (338 bp) in lane 5 and ST7 (650 bp) in lane 6; b) in poultry showing positive bands of ST1 (351 bp) in lane 1, ST2 (704 bp) in lane 2, ST6 (338 bp) in lane 3, and ST7 (650 bp) in lane 4; and c) in humans showing positive bands of ST1 (351 bp) in lane 1, ST2 (704 bp) in lane 2, ST3 (526 bp) in lane 3, ST4 (487 bp) in lane 4. M is the molecular weight marker at 100-bp.

\section{DISCUSSION}

Several factors suggested the zoonotic potential of blastocystosis. These include; its high frequency in animals and animal handlers with morphologically indistinguishable parasites $^{[26-29]}$, and the detection of identical or highly similar genetic STs of the parasite in both humans and a wide range of animals ${ }^{[12,21,30]}$. Thus, it has been proposed that human infections may result from zoonotic transmission of the parasite. In Egypt, there is limited data on Blastocystis STs, especially in non-human hosts. In this study, we investigated the occurrence and ST distribution of Blastocystis spp. isolated from various nonhuman hosts (domestic mammals and poultry) and their incontact humans.

The occurrence of Blastocystis spp. among poultry in the present study was $69.8 \%$. Similarly, high prevalence rates were detected among domestic fowls and ostriches (100\%) in Japan ${ }^{[31]}$, free-range chicken $(80-100 \%)$ in Malaysia ${ }^{[32]}$, and other bird species $(90 \%)$ from Colombia ${ }^{[33]}$. Moderate prevalence rate $(27 \%)$ was reported from a poultry center in Pakistan ${ }^{[29]}$. The scavenging habits of birds, especially those reared in rural areas where birds are left free-ranging 
for feeding, increased the likelihood of acquiring the infection from the environment. Also, the drinking water pots used for these birds are usually contaminated with their feces, which presents a chance of spreading the infection to the same or other species of birds reared together, which is also common in rural areas. Transmission of Blastocystis infection was proven to occur easily between the same or different bird species ${ }^{[34]}$.

With regards to Blastocystis infection in the domestic mammal species examined, it was $72.2 \%$ in cattle, $28.6 \%$ in goats, $25 \%$ in camels and $7.1 \%$ in donkeys. These findings are comparable with other studies reporting blastocystosis in $71 \%$ of cattle in Japan ${ }^{[28]}$, in $30.9 \%$ of goats in Malaysia ${ }^{[35]}$, in $24 \%$ of camels in Libya ${ }^{[10]}$ and in $12.5 \%$ of horses in Thailand ${ }^{[36]}$. However, a lower percentage was reported in cattle from the UK $(22.5 \%)$. Furthermore, the infection was not reported in cattle samples from France and Italy, or in donkey samples from Libya and the $\mathrm{UK}^{[10]}$ and in goat samples from Japan ${ }^{[28]}$. These differences may be attributed to a difference in the number of samples examined, different diagnostic techniques, different management systems in farms and barns owned by farmers or different adaptation by animals ${ }^{[37]}$.

In this study, the occurrence of Blastocystis spp. among humans in contact with animals was $35.7 \%$, which was in accordance with previous reports of human blastocystosis in Egypt, regardless of the state of contact with animals. A prevalence of $22.4 \%$ was reported among subjects from Dakahlia governorate ${ }^{[38]}$, while it was $33.3 \%$ among patients complaining of GIT symptoms ${ }^{[39]}$ and $26.5 \%$ among pregnant women ${ }^{[40]}$. The situation was quite similar in other developing countries: a prevalence of $41 \%$ was reported among animal handlers from Malaysia ${ }^{[27]}, 26.1 \%$ in subjects from rural communities in $\mathrm{Nepa}^{[41]}$ and $37.2 \%$ in Thailand ${ }^{[42]}$. Generally, prevalence of Blastocystis spp. infection appears to be higher in developing countries than in developed countries. This could be attributed to several risk factors including poor community sanitation and/or low level of care to environmental hygiene, the absence of potable water and adequate elimination of feces, socioeconomic status, poor personal hygiene and high-risk behavior $^{[43]}$.

In this study, we used seven sets of STS primers for the amplification of ST1-ST7. This method does not enable the detection of other STs (ST8-ST17). The use of STS primers was proven advantageous for epidemiological studies and for resource-limited countries ${ }^{[44]}$. Also, it allows the detection of MSI involving ST1-ST7 that are not detected by sequencing and are therefore greatly underestimated in most data sets derived from sequencing ${ }^{[10]}$. Moreover, this method allows the identification of samples worthy of further investigation. Such samples include Blastocystis isolates that cannot be typed by STS primers and isolates with shared STs in both humans and animals that could support the zoonotic potential of this parasite. However, with the use of STS primers, it should be noted that some mixed infections could be missed if they involve ST8ST17. Also, some ST4 infections could be under diagnosed by the STS method, especially when mixed with other STs, due to genetic variation within this $\mathrm{ST}^{[45]}$.

Among the domestic bird species examined, ST7 predominated $(77.8 \%)$, followed by ST6 (17.8\%) indicating that a strong association between these STs and avian hosts exists ${ }^{[8,12]}$. Conversely, ST1 and ST2 infections were reported less frequently ( $7.8 \%$ and $1.1 \%$, respectively) in domestic birds. These STs were also reported previously among chicken in Japan $^{[46]}$. ST6 was reported as the only detected ST among 52 samples from different bird species $^{[33]}$, which could be attributed to geographically restricted distribution ${ }^{[12]}$.

As for domestic mammals, we observed the occurrence of ST1 and STs 3-7 among infected mammals' samples, but ST2 was not detected. Previous studies reported ST2 mainly from humans and non-human primates ${ }^{[12]}$, and only few isolates from pigs, dogs, rodents and a chicken ${ }^{[20,33,37,46]}$. In the present study, ST4 infection was detected in four animals (two camels and two goats) with MSI. Rodents were previously proposed as a reservoir of ST4 as this ST was mostly isolated from different rodent species. However, Alfellani et al. ${ }^{[10]}$ could not find ST4 in any of the rodent hosts screened. Infection of camels with ST4 has not been reported previously, although it was reported from a buffalo, a goat and two pigs ${ }^{[22]}$. In this study, we detected ST6 and ST7 in camels with MSI. These STs were detected in goats with MSI using STS primers ${ }^{[35]}$. Alfellani et al. ${ }^{[10]}$, using 'barcoding' of the small-subunit rRNA gene, did not find these STs in camels which could have been missed if they had been integrated with MSI. Detection of these STs (ST6 and ST7) in domestic mammals, giving the high incidence in avian hosts, could be due to crosstransmission occurring between host species, as domestic animals are often kept in close proximity to each other in rural areas. In this study, six samples isolated from cattle could not be typed with STS primers used. Recently, ST10 was the most common ST detected in cattle samples from four countries (Denmark, Libya, the UK, and the USA), proving that the distribution of this ST has no geographic restriction. Also, ST14 was reported from cattle samples from Libya and the USA in the same study ${ }^{[10]}$. Subsequent sequence analysis of isolates that could not be typed may reveal the underlying subtype in these isolates.

In our study, four Blastocystis STs (ST1-ST4) were identified from humans in close contact with animals with a predominance of ST3 $(60.7 \%)$, followed by ST1 (35.7\%), ST2 (7.1\%) and then ST4 (5.4\%). Previous studies in Egypt either reported three STs (ST1-ST3) $)^{[3,47]}$ or four STs (ST1, ST3, ST6, and ST7) ${ }^{[48]}$. Among these previous studies, ST3 predominance ranged from 55-62\%. In contrast, ST4 has not been reported previously. In our results, all human isolates with ST4 infection had animals 
infected with the same ST. Similarly, ST4 was detected in a buffalo and a pig and in their respective owner suggesting the zoonotic potential of Blastocystis spp. ST4 ${ }^{[22,41]}$. In contrast, in the present study, a minority of individuals with ST1 (40\%) and ST3 (14.7\%), and none of those with ST2 showed ST similarities with the detected Blastocystis STs in their domestic mammals and/or birds, suggesting that direct handling of animals is not a major contributor to human infections with Blastocystis in Egypt. In keeping with our results, two Libyan studies demonstrated that ST1 and ST3 predominated among Libyan population (89\%), while other STs (ST5 and ST10) predominated in livestock (50\%). Nevertheless, ST1 and ST3 were detected in Libyan animals at minor percentages $(7 \%$ and $9 \%$, respectively) suggesting that human blastocystosis results mainly from anthroponotic transmission ${ }^{[10,49]}$. Related to this, several studies have implicated contaminated water as a source of Blastocystis infection, carried from infected human or animal feces. In China, consumption of water plants and drinking non boiled water were considered risk factors for infections with ST1 and ST $3^{[50]}$. Also, ST1 and ST4 were detected in two rivers in Nepal[22]. Moreover, viable cysts of Blastocystis were detected in treated water samples, indicating the resistance of cysts to treatment processes $^{[51]}$. Transmission may also occur through other surrounding animals (e.g. stray dogs and cats) prevalent in rural areas where there is an inadequate elimination of feces from streets, and maybe from harmful rodents eating crops and spreading infection through their feces. Recently, Blastocystis infection was detected in $24 \%$ of stray dogs from India with a diverse range of STs $(\mathrm{ST} 1,4,5 \text { and } 6)^{[52]}$. Therefore, future studies need to be conducted to identify other potential risks of transmission to humans with different Blastocystis STs in Egypt, including water sources as well as other potential reservoir animals.

\section{CONCLUSION}

Blastocystis spp. was commonly detected in both humans and domestic animals in the present study with varying detection rates. All seven STs tested by the STS primers were detected in the infected animals examined in the present study, highlighting the broad genetic diversity observed among Blastocystis spp. isolated from animals. Only four STs (ST1-ST4) were identified among humans, suggesting that these STs can easily infect humans, and the animals carrying the same STs could be possible reservoirs. However, the predominance of ST3 in humans and the detection of specific STs (ST5-ST7) in animals but not in humans highlighted the potential role of host specificity in restricting the range of zoonotic transmission ${ }^{[53]}$. In addition, the use of multi-locus sequence typing demonstrated that variation within ST3 is much wider among non-human primates than in humans concluding that a shared ST alone may be too rough a criterion to link between human and non-human infections ${ }^{[54]}$. Further genomic studies are required for analysis of the shared STs in humans and animals to confirm the zoonotic potential of these isolates by identifying the same strain in both hosts and not just the same ST.

Author contribution: AB Mokhtar proposed the research idea, planned the study design, shared in the laboratory work and wrote the manuscript. AI Youssef collected the study samples and shared in the laboratory work.

\section{ACKNOWLEDGMENT}

The authors would like to acknowledge Dr. Sherif M Abaza, Professor of Parasitology, Faculty of Medicine, Suez Canal University for his kind assistance in revising the manuscript.

\section{FUNDING}

This research did not receive any specific grant from funding agencies in the public, commercial, or not-forprofit sectors.

\section{CONFLICT OF INTEREST}

None.

\section{REFERENCES}

1. Yoshikawa H, Wu Z, Howe J, Hashimoto T, GeokChoo N, Tan KS. Ultrastructural and phylogenetic studies on Blastocystis isolates from cockroaches. J Eukaryot Microbiol, 2007; 54:33-37.

2. Tan KS. New Insights on classification, identification, and clinical relevance of Blastocystis spp. Clin Microbiol Rev, 2008; 21: 639-665.

3. Souppart L, Moussa H, Cian A, Sanciu G, Poirier P, El-Alaoui H, et al. Subtype analysis of Blastocystis isolates from symptomatic patients in Egypt. Parasitol Res, 2010; 106:505-511.

4. Zhang X, Qiao JY, Zhou XJ, Yao FR, Wei ZC. Morphology and reproductive mode of Blastocystis hominis in diarrhea and in vitro. Parasitol Res, 2007; 101:43-51.

5. El-Gebaly NSM, Zaki MM. Ultrastuctural Intestinal pathology induced by human Blastocystis in experimentally infected mice. PUJ, 2012; 5(2):127134. 
6. Yoshikawa H, Yoshida K, Nakajima A, Yamanri K, Iwatani S, Kimata M. Feco-oral transmission of the cyst form of Blastocystis hominis in rats. Parasitol Res, 2004; 94:361-366.

7. Yan Y, Su S, Ye J, Lai X, Lai R, Liao H, et al. Blastocystis sp. subtype 5: a possibly zoonotic genotype. Parasitol Res, 2007; 101:1527-1532.

8. Noël C, Dufernez F, Gerbod D, Edgcomb V, DelgadoViscogliosi P, Ho L, et al. Molecular phylogenies of Blastocystis hominis isolates from different hosts: implications of genetic diversity, identification of species, and zoonosis. J Clin Microbiol, 2005; 43:348-355.

9. Ithoi I, Jali A, Mak JW, Sulaiman WY, Mahmud R. Occurrence of Blastocystis in water of two rivers from recreational areas in Malaysia. J Parasitol Res, 2011; 2011:123916.

10. Alfellani MA, Taner-Mulla D, Jacob AS, Imeede CA, Yoshikawa H, Stensvold CR, et al. Genetic diversity of Blastocystis in livestock and zoo animals. Protist, 2013; 164:497-509.

11. Özyurt M, Kurt O, Mølbak K, Nielsen HV, Haznedaroglu T, Stensvold CR. Molecular epidemiology of Blastocystis infections in Turkey. Parasitol Int, 2008; 57:300-306.

12. Stensvold CR, Alfellani MA, Nørskov-Lauritsen S, Prip K, Victory EL, Maddox C, et al. Subtype distribution of Blastocystis isolates from synanthropic and zoo animals and identification of a new subtype. Int J Parasitol, 2009; 39:473-479.

13. Li LH, Zhang XP, Lv S, Zhang L, Yoshikawa H, $\mathrm{Wu} \mathrm{Z}$, et al. Cross-sectional surveys and subtype classification of human Blastocystis isolates from four epidemiological settings in China. Parasitol Res, 2007; 102:83-90.

14. Souppart L, Sanciu G, Cian A, Wawrzyniak I, Delbac F, Capron M, et al. Molecular epidemiology of human Blastocystis isolates in France. Parasitol Res, 2009; 105:3-17.

15. Yakoob J, Jafri W, Asim Beg M, Abbas Z, Naz S, Islam $\mathrm{M}$, et al. Irritable bowel syndrome: is it associated with genotypes of Blastocystis hominis. Parasitol Res, 2010; 106:1033-1038

16. Meloni D, Sanciu G, Poirier P, El Alaoui H, Chabé M, Delhaes L, et al. Molecular subtyping of Blastocystis sp. Isolates from symptomatic patients in Italy. Parasitol Res, 2011; 109:613-619.
17. Roberts T, Stark D, Harkness J, Ellis J. Subtype distribution of Blastocystis isolates identified in a Sydney population and pathogenic potential of Blastocystis. Eur J Clin Microbiol Infect Dis, 2013; 32:335-343.

18. Cian A, El Safadi D, Osman M, Moriniere R, Gantois N, Benamrouz-Vanneste S, et al. Molecular epidemiology of Blastocystis sp. in various animal groups from two French zoos and evaluation of potential zoonotic risk. PLoS One, 2017; 12:e0169659.

19. Parkar U, Traub J, Kumar S, Mungthin M, Vitali $\mathrm{S}$, Leelayoova S, et al. Direct characterization of Blastocystis from faeces by PCR and evidence of zoonotic potential. Parasitol, 2007; 134:359-367.

20. Eroglu F, Koltas IS. Evaluation of the transmission mode of $B$. hominis by using PCR method. Parasitol Res, 2010; 107:841-845.

21. Parkar U, Traub RJ, Vitali S, Elliot A, Levecke B, Robertson I, et al. Molecular characterization of Blastocystis isolates from zoo animals and their animal-keepers. Vet Parasitol, 2010; 169:8-17.

22. Lee IL, Tan TC, Karmacharya BM, Suresh KG Blastocystis sp.: waterborne zoonotic organism, a possibility? Parasit Vectors, 2012; 5:130-135.

23. Jones WR. The experimental infection of rats with Entamoeba histolytica with a method for evaluating the anti-amoeboic properties of new compounds. Ann Trop Med Parasitol, 1946; 40:130-140.

24. Yoshikawa $\mathrm{H}, \mathrm{Wu} \mathrm{Z}$, Kimata $\mathrm{M}$, Iseki I, Ali M, Hossain B, et al. Polymerase chain reaction-based genotype classification among human Blastocystis hominis population isolated from different countries. Parasitol Res, 2004; 92:22-29.

25. Stensvold CR, Suresh GK, Tan KSW, Thompson RCA, Traub RJ, Viscogliosi E, et al. Terminology for Blastocystis subtypes - a consensus. Trends Parasitol, 2007; 23:93-96.

26. Lee MG, Stenzel DJ. A survey of Blastocystis in domestic chickens. Parasitol Res, 1999; 85:109 - 117.

27. Salim HR, Kumar GS, Vellayan S, Mak JW, Anuar $\mathrm{AK}$, Init I, et al. Blastocystis in animal handlers. Parasitol Res, 1999; 85:1032-1033.

28. Abe N, Nagoshi M, Takami K, Sawano Y, Yoshikawa $\mathrm{H}$. A survey of Blastocystis sp. in livestock, pets and zoo animals in Japan. Vet Parasitol, 2002; 106:203212 . 
29. Haider SS, Baqai R. Detection of Blastocystis hominis in humans and poultry. Infect Dis J Pakistan, 2008; $17: 43-47$.

30. Yoshikawa H, Wu Z, Pandey K, Pandey BD, Sherchand $\mathrm{JB}$, Yanagi $\mathrm{T}$, et al. Molecular characterization of Blastocystis isolates from children and rhesus monkeys in Kathmandu, Nepal. Vet Parasitol, 2009; 160:295-300.

31. Yamada M, Yoshikawa H, Tegoshi T, Matsumoto Y, Yoshikawa T, Shiota T, et al. Light microscopical study of Blastocystis spp. in monkeys and fowls. Parasitol Res, 1987; 73:527-531.

32. Haziqah FMT, Thani CP, Zain MSN, Kumar SG, Hemalatha C, Premaalatha B. A preliminary study of Blastocystis sp. isolated from chicken in Perak and Selangor, Malaysia. Malays J Vet Res, 2014; 5:21-25.

33. Ramírez JD, Sanchez LV, Bautista DC, Corredor AF, Florez AC, Stensvold CR. Blastocystis subtypes detected in humans and animals from Colombia. Infect Genet Evol, 2014; 22:223-228.

34. Tanizaki A, Yoshikawa H, Iwatani S, Kimata I. Infectivity of Blastocystis isolates from chickens, quails and geese in chickens. Parasitol Res, 2005; 96:57-61.

35. Tan TC, Tan PC, Sharma R, Sugnaseelan S, Suresh KG. Genetic diversity of caprine Blastocystis from Peninsular Malaysia. Parasitol Res, 2013; 112:85-89.

36. Thathaisong U, Worapong J, Mungthin M, Tan-Ariya P, Viputtigul K, Sudatis A, et al. Blastocystis isolates from a pig and a horse are closely related to Blastocystis hominis. J Clin Microbiol, 2003; 41:967-975.

37. Navarro C, Domínguez-Márquez MV, Garijo-Toledo MM, Vega-García S, Fernández-Barredo S, PérezGracia MT, et al. High prevalence of Blastocystis sp. in pigs reared under intensive growing systems: frequency of ribotypes and associated risk factors. Vet Parasitol, 2008; 153:347-358.

38. El-Shazly AM, Awad SE, Sultan DM, Sadek GS, Khalil HH, Morsy TA. Intestinal parasites in Dakahlia Governorate, with different techniques in diagnosing protozoa. J Egypt Soc Parasitol, 2006; 36:1023-34.

39. Rayan HZ, Ismail OA, El-Gayar EK. Prevalence and clinical significance of Dientamoeba fragilis infections in patients suspected to have intestinal parasitic infection. J Egypt Soc Parasitol, 2007; 37:599-608.

40. El-Deeb HK, Salah-Eldin H, Khodeer S. Blastocystis hominis as a contributing risk factor for development of iron deficiency anaemia in pregnant women. Parasitol Res, 2012; 110:2167-174.

41. Lee IL, Tan TC, Tan PC, Nanthiney DR, Biraj MK, Surendra KM, et al. Predominance of Blastocystis sp. subtype 4 in rural communities, Nepal. Parasitol Res, 2012; 110:1553-1562.

42. Popruk S, Udonsom R, Koompapong K, Mahittikorn A, Kusolsuk T, Ruangsittichai J, et al. Subtype distribution of Blastocystis in Thai-Myanmar border, Thailand. Korean J Parasitol. 2015; 53:13-19.

43. Yoshikawa H. Epidemiology, transmission, and zoonotic potential of Blastocystis in human and animals, in Melhorn H, Tan KSW, Yoshikawa H (Eds.), Blastocystis: pathogen or passenger? Heidelberg: Springer 2012; 37-49.

44. Belleza ML, Reyes JC, Tongol-Rivera PN, Rivera WL. Subtype analysis of Blastocystis sp. isolates from human and canine hosts in an urban community in the Philippines. Parasitol Int, 2016; 65:291-294.

45. Stensvold CR. Comparison of sequencing (barcode region) and sequence-tagged-site PCR for Blastocystis subtyping. J Clin Microbiol, 2013; 51:190-194.

46. Yoshikawa H, Abe N, Wu Z. PCR-based identification of zoonotic isolates of Blastocystis from mammals and birds. Microbiol, 2004; 150:1147-1151.

47. Abaza SM, Rayan HZ, Soliman RH, Nemr NA, Mokhtar AB. Subtype analysis of Blastocystis spp. isolates from symptomatic and asymptomatic patients in Suez Canal University Hospitals, Ismailia, Egypt. PUJ, 2014; 7:56-67.

48. Hussein EM, Hussein AM, Eida MM, Atwa MM. Pathophysiological variability of different genotypes of human Blastocystis hominis Egyptian isolates in experimentally infected rats. Parasitol Res, 2008; 102:853-860.

49. Alfellani MA, Stensvold CR, Vidal-Lapiedra A, Onuoha ES, Fagbenro-Beyioku AF, Clark CG. Variable geographic distribution of Blastocystis subtypes and its potential implications. Acta Trop, 2013; 126:11-18.

50. Li LH, Zhou XN, Du ZW, Wang XZ, Wang LB, Jiang JY, et al. Molecular epidemiology of human Blastocystis in a village in Yunnan province, China. Parasitol Int, 2007; 56:281-286.

51. Banaticla JE, Rivera WL. Detection and subtype identification of Blastocystis isolates from waste water samples in the Philippines. J Water Health, 2011; 9:128-37. 
52. Wang W, Cuttell L, Bielefeldt-Ohmann H, Inpankaew T, Owen H, Traub RJ. Diversity of Blastocystis subtypes in dogs in different geographical settings. Parasites Vect, 2013; 6:215-220.

53. Yoshikawa $H$, Tokoro $M$, Nagamoto $T$, Arayama $\mathrm{S}$, Asih PBS, Rozi IE, et al. Molecular survey of
Blastocystis sp. from humans and associated animals in an Indonesian community with poor hygiene. Parasitol Int, 2016; 65:780-784.

54. Stensvold CR, Alfellani M, Clark CG. Levels of genetic diversity vary dramatically between Blastocystis subtypes. Infect Genet Evol, 2012; 12:263-273. 